\title{
Q-balls constructed of spinors in Lagrangians with $S U(2)$ symmetry
}

\author{
Athanasios Prikas \\ Physics Department, National Technical University, Zografou Campus, \\ 15780 Athens, Greece. ${ }^{1}$
}

\begin{abstract}
In the present work we investigate the existence and stability properties of q-balls which consist of a couple of scalar fields, forming an $S U(2)$ doublet in a Lagrangian with a global $S U(2)$ symmetry. We find that these spinors can form a localized and stable field configuration, if they rotate in their internal $S U(2)$ space. We find the energy and charge of the soliton in both thin and thick-wall approximation and we prove its stability against decaying to free particles. We also find the asymptotic forms of the scalar and gauge field and the energy and charge of the configuration when the $S U(2)$ symmetry is local. The only assumption is the smallness of the coupling constant $g$. Using numerical methods we prove the stability of the q-ball in the local case.
\end{abstract}

PACS number(s): 11.27.+d, 11.10.Lm

\footnotetext{
${ }^{1}$ e-mail: aprikas@central.ntua.gr
} 


\section{Introduction}

Q-balls are non-topological solitons appearing in Lagrangians of scalar fields with a global $U(1)$ or $S O(2)$ symmetry. Though they appeared as a rather mathematical object [1], through a series of papers they revealed their physical properties [2, 3, 4. Q-balls can be observed at, or near, the minimum of the $\sqrt{U /(|\Phi|)^{2}}$ quantity, where $U$ is the potential and $\Phi$ is the scalar field. At the above minimum one can prove that the energy is minimized with respect to the charge. The minimum of this quantity should be less than the particle mass, in order the soliton to be stable. Much more interest was concentrated on the subject when their possible existence in supersymmetric extensions of the Standard Model was considered [5, 6, 7, 8, where the $U(1)$ charge is for example the baryon number of the supersymmetric partners of baryons. Flat $U(1)$ directions in the potential of such extensions can offer a plausible explanation to the problem of baryogenesis ([9, 10]).

Another type of q-ball type solutions has been studied, namely the socalled non-abelian q-balls [11, 12] where we have scalar fields in a Lagrangian with a global $S U(3)$ or $S O(3)$ symmetry usually and the fields belong to the adjoint representation of the symmetry group. Such particles may correspond to the sgauginos in $N=2$ supersymmetric theories. Also, q-balls with a local $U(1)$ symmetry have been studied in [13].

In a recent series of papers [14, 15, 16, a great number of $S U(2)$ flat directions in the superpotential of the Minimal Supersymmetric Standard Model and a rich phenomenology concerning them have been investigated. So the plausible question is to ask if a new type of q-balls can appear in such directions, enriching the particle phenomenology. In the present work we try to do the following three things: a)We present the general formalism concerning q-balls when the fields transform according to the doublet representation of the symmetry group because this case has not been studied and may turn out to be useful in the search of q-solitons composed of supersymmetric partners of the usual leptons. b)We present the formalism of gauged q-balls which describe scalar particles with "weak" interactions. c)We fully investigate the properties of the above solitons both analytically and numerically in the so-called thin- and thick-wall approximation. Our main interest is to verify their stability when varying the gauge coupling, the frequency with which the soliton field rotates within its internal $S U(2)$ space, or other phenomenological parameters. 


\section{Global SU(2) symmetry. Thin-wall approximation}

We regard a Lagrangian

$$
L=\partial_{\mu} \vec{\varphi}^{\dagger} \partial^{\mu} \vec{\varphi}-U\left(\vec{\varphi}^{\dagger} \vec{\varphi}\right)
$$

characterized by a global $S U(2)$ symmetry concerning the couple of scalar fields:

$$
\vec{\varphi}=\left(\begin{array}{c}
\varphi_{1} \\
\varphi_{2}
\end{array}\right)
$$

The energy is provided by the equation

$$
\mathrm{E}=\int d^{3} x\left(\nabla \vec{\varphi}^{\dagger} \nabla \vec{\varphi}+\dot{\vec{\varphi}}^{\dagger} \dot{\vec{\varphi}}+U\right)
$$

The currents are three as the generators of the algebra considered. These currents are

$$
j_{\alpha}^{0}=\left(\begin{array}{cc}
\dot{\vec{\varphi}}^{\dagger} & \dot{\vec{\varphi}}^{T}
\end{array}\right)\left(\begin{array}{cc}
\frac{\imath}{2} \tau_{\alpha} & 0 \\
0 & -\frac{\imath}{2} \tau_{\alpha}^{*}
\end{array}\right)\left(\begin{array}{c}
\vec{\varphi} \\
\vec{\varphi}^{*}
\end{array}\right)
$$

where $\tau_{\alpha}$ are the Pauli matrices:

$$
\tau_{1}=\left(\begin{array}{cc}
0 & 1 \\
1 & 0
\end{array}\right) \quad \tau_{2}=\left(\begin{array}{cc}
0 & -\imath \\
\imath & 0
\end{array}\right) \quad \tau_{3}=\left(\begin{array}{cc}
1 & 0 \\
0 & -1
\end{array}\right) .
$$

We choose

$$
\vec{\varphi}(\vec{r}, t)=\exp \left(\frac{\imath}{2} \tau_{\alpha} \varepsilon_{\alpha} t\right) \vec{\sigma}(\vec{r})
$$

In the so-called thin-wall approximation we choose $\vec{\sigma}(\vec{r})$ a step-function, with a constant value within a volume $V$ and zero outside. We also choose a spherically symmetric configuration, i.e. $\vec{\sigma}(\vec{r})=\vec{\sigma}(r)$ so as to minimize the contribution of the surface effects to the total energy. So the energy can be written

$$
\mathrm{E}=\dot{\vec{\varphi}}^{\dagger} \dot{\vec{\varphi}} V+U V
$$

and using the chosen field configuration:

$$
\mathrm{E}=\frac{1}{4}\left(\varepsilon_{1}^{2}+\varepsilon_{2}^{2}+\varepsilon_{3}^{2}\right) \vec{\sigma}^{\dagger} \vec{\sigma} V+U V .
$$


We can now define the charges in the above thin-wall approximation where we take

$$
Q_{i}=\frac{1}{2} \varepsilon_{i} \vec{\sigma}^{\dagger} \vec{\sigma} V
$$

where the definitions:

$$
Q_{\alpha}=\int d^{3} x j_{\alpha}^{0}
$$

and

$$
\mathbf{Q} \equiv\left(Q_{1}, Q_{2}, Q_{3}\right)
$$

hold. We now want to write energy as a function of the charges. It is a matter of simple algebra to show that:

$$
\mathrm{E}=\frac{\mathrm{Q}^{2}}{\vec{\sigma}^{\dagger} \vec{\sigma} V}+U V
$$

Regarding energy as a function of the volume and minimizing it with respect to the volume, we take that the minimum is at:

$$
V=\sqrt{\frac{\mathrm{Q}^{2}}{\vec{\sigma}^{\dagger} \vec{\sigma} U}}
$$

and the energy takes the form:

$$
\mathrm{E}=\sqrt{\mathrm{Q}^{2}} \sqrt{\frac{4 U}{\vec{\sigma}^{\dagger} \vec{\sigma}}}
$$

The energy of the free particles carrying charge $\sqrt{\mathbf{Q}^{2}}$ is $2 m \cdot \sqrt{\mathbf{Q}^{2}}$. (It is a matter of simple algebra to prove this. The physical interpretation is obvious because we now have two particles, each with mass $m$.) So if the quantity $\left(U / \vec{\sigma}^{\dagger} \vec{\sigma}\right)^{1 / 2}$ is less than the mass of the free particles then the energy of the (14) field configuration is less than the energy of the free waves and the soliton is preserved stable against decaying into free particles. We know that $\sqrt{\mathrm{Q}^{2}}$, quantity is both conserved, as every single current is conserved, and $S U(2)$ invariant. We now investigate a potential of fourth power in $|\varphi| \equiv\left(\vec{\varphi}^{\dagger} \vec{\varphi}\right)^{1 / 2}$, namely:

$$
U=m^{2}|\varphi|^{2}-\frac{2 \alpha}{3}|\varphi|^{3}+\frac{\beta}{2}|\varphi|^{4} .
$$

This is the more general potential of fourth order in the fields. The theory is non-renormalizable but this is not a serious problem as we can regard the 
Lagrangian as a phenomenological one. This potential can be proved very useful in a supersymmetric theory. Making the rescallings $|\varphi| \rightarrow\left(m^{2} / \alpha\right)|\varphi|$, $x \rightarrow x / m$ and defining $B \equiv \beta m^{2} / \alpha$ we take

$$
U=|\varphi|^{2}-\frac{2}{3}|\varphi|^{3}+\frac{B}{2}|\varphi|^{4} .
$$

Minimizing the $\left(4 U /|\varphi|^{2}\right)^{1 / 2}$ quantity with respect to the field and calling it $\sqrt{\varepsilon_{0}^{2}}$ we can take that the minimum is at

$$
|\varphi|=2 / 3 B
$$

Then:

$$
\begin{gathered}
\sqrt{\varepsilon_{0}^{2}}=2 \sqrt{1-2 / 9 B}, \\
\mathbf{Q}=(1 / 2)(2 / 3 B)^{2} V \boldsymbol{\varepsilon}, \\
\mathrm{E}=(2 / 3 B)^{2} V \sqrt{\varepsilon_{0}^{2}} \sqrt{1-2 / 9 B},
\end{gathered}
$$

where $\boldsymbol{\varepsilon} \equiv\left(\varepsilon_{1}, \varepsilon_{2}, \varepsilon_{3}\right)$. It is easy then to verify that $\mathrm{E}=\sqrt{\varepsilon_{0}^{2}} \sqrt{\mathbf{Q}^{2}}$.

\section{Local SU(2) case. General setting}

The Lagrangian for the case with a local $S U(2)$ symmetry is written

$$
L=\vec{\varphi}^{\dagger}\left(\overleftarrow{\partial}^{\mu}+\imath g \frac{\boldsymbol{\tau} \cdot \mathbf{A}^{\mu}}{2}\right)\left(\partial_{\mu}-\imath g \frac{\boldsymbol{\tau} \cdot \mathbf{A}_{\mu}}{2}\right) \vec{\varphi}-U\left(\vec{\varphi}^{\dagger} \vec{\varphi}\right)-\frac{1}{4} \mathbf{F}^{\mu \nu} \mathbf{F}_{\mu \nu}
$$

where:

$$
\mathbf{F}^{\mu \nu}=\partial^{\mu} \mathbf{A}^{\nu}-\partial^{\nu} \mathbf{A}^{\mu}+g \mathbf{A}^{\mu} \times \mathbf{A}^{\nu} .
$$

and $g$ is the coupling constant.

We now regard: a)Spherically symmetric fields in order to minimize the contribution to the total energy of the so-called surface effects. b)Static gauge fields. c) Only the zero component of the gauge field differing from zero, the other components being equal to zero. This is not a gauge-fixing but a statement that the gauge field configuration is static and, consequently, the magnetic fields absent. d) $\mathbf{A}_{0}$ finite everywhere and zero at infinity. If we also use the initial ansatz for the scalar field we take for the Lagrangian:

$$
L=\frac{1}{4} \vec{\sigma}^{\dagger}\left(\varepsilon-g \mathbf{A}_{0}\right)^{2} \vec{\sigma}-U\left(\vec{\sigma}^{\dagger} \vec{\sigma}\right)+\frac{1}{2}\left(\partial_{a} \mathbf{A}_{0}\right)^{2}+\left(\partial_{a} \vec{\sigma}^{\dagger}\right)\left(\partial^{a} \vec{\sigma}\right),
$$


where Latin indices run from 1 to 3 and Greek indices run from 0 to 3 .

Defining

$$
\boldsymbol{\theta}=\boldsymbol{\varepsilon}-g \mathbf{A}_{0},
$$

regarding $\varepsilon$ as a constant and after a little algebra we take

$$
\mathrm{L}=4 \pi \int r^{2} d r\left[\frac{1}{4} \vec{\sigma}^{\dagger} \vec{\sigma} \boldsymbol{\theta}^{2}-U\left(\vec{\sigma}^{\dagger} \vec{\sigma}\right)+\frac{1}{2 g^{2}} \boldsymbol{\theta}^{\prime 2}-\vec{\sigma}^{\dagger^{\prime}} \vec{\sigma}^{\prime}\right],
$$

where the prime denotes the derivative with respect to the radius $r$ and the last term can be absent if $\vec{\sigma}(r)$ is a step-function. The Euler-Lagrange equations for the above fields $\vec{\sigma}, \boldsymbol{\theta}$, are

$$
\begin{gathered}
\vec{\sigma}^{\prime \prime}+\frac{2}{r} \vec{\sigma}^{\prime}+\frac{1}{4} \boldsymbol{\theta}^{2} \vec{\sigma}-\frac{\partial U}{\partial \vec{\sigma}^{\dagger}}=0, \\
\boldsymbol{\theta}^{\prime \prime}+\frac{2}{r} \boldsymbol{\theta}^{\prime}-\frac{1}{2} g^{2}\left(\vec{\sigma}^{\dagger} \vec{\sigma}\right) \boldsymbol{\theta}=0 .
\end{gathered}
$$

The total energy of the field configuration can be written

$$
\mathrm{E}=4 \pi \int r^{2} d r\left[\frac{1}{4} \vec{\sigma}^{\dagger} \vec{\sigma} \boldsymbol{\theta}^{2}+U\left(\vec{\sigma}^{\dagger} \vec{\sigma}\right)+\frac{1}{2 g^{2}} \boldsymbol{\theta}^{\prime 2}+\vec{\sigma}^{\dagger^{\prime}} \vec{\sigma}^{\prime}\right] .
$$

Now we will find the total charge. A component of the current is defined as:

$$
j_{\alpha 0}=\left[\begin{array}{ll}
\frac{\partial L}{\partial\left(\partial_{0} \vec{\varphi}\right)} & \frac{\partial L}{\partial\left(\partial_{0} \vec{\varphi}^{*}\right)}
\end{array}\right]\left[\begin{array}{cc}
\frac{\imath}{2} \tau_{\alpha} & 0 \\
0 & -\frac{2}{2} \tau_{\alpha}^{*}
\end{array}\right]\left[\begin{array}{c}
\vec{\varphi} \\
\vec{\varphi}^{*}
\end{array}\right],
$$

which in the special case of the local symmetry can be written

$$
j_{\alpha 0}=\left[\vec{\varphi}^{\dagger}\left(\overleftarrow{\partial}_{0}+\imath g \frac{\boldsymbol{\tau} \cdot \mathbf{A}_{0}}{2}\right), \quad \vec{\varphi}^{T}\left(\partial_{0}-\imath g \frac{\tau^{*} \cdot \mathbf{A}_{0}}{2}\right)\right]\left[\begin{array}{cc}
\frac{\imath}{2} \tau_{\alpha} & 0 \\
0 & -\frac{\imath}{2} \tau_{\alpha}^{*}
\end{array}\right]\left[\begin{array}{c}
\vec{\varphi} \\
\vec{\varphi}^{*}
\end{array}\right] .
$$

After some algebra we take

$$
Q_{i}=2 \pi \int r^{2} d r \theta_{i} \vec{\sigma}^{\dagger} \vec{\sigma}
$$

Another useful relation can be obtained from equation 27.

$$
\left(r^{2} \boldsymbol{\theta}^{\prime}\right)^{\prime}=\frac{1}{2} g^{2}\left(\vec{\sigma}^{\dagger} \vec{\sigma}\right) \boldsymbol{\theta} r^{2} .
$$


By the definition of $\boldsymbol{\theta}$ and by substituting eq. 31 in 30 we can take an asymptotic relation connecting $\theta_{i}$, or equivalently $A_{i 0}$ and $Q_{i}$, when $r \rightarrow \infty$. The relation is:

$$
\theta_{i}=\varepsilon_{i}-g^{2} \frac{Q_{i}}{4 \pi r} .
$$

Also for large $r, \vec{\sigma}$ is small, so as to have a localized solution of the equations of motion, so $U\left(\vec{\sigma}^{\dagger} \vec{\sigma}\right) \cong m^{2} \vec{\sigma}^{\dagger} \vec{\sigma}$ and equation (26) takes the form:

$$
\vec{\sigma}^{\prime \prime}+\frac{2}{r} \vec{\sigma}^{\prime}+\vec{\sigma}\left(\frac{1}{4} \varepsilon^{2}-m^{2}\right)=0
$$

the solution of which has the asymptotic form: $\vec{\sigma}_{i} \propto \exp \left(-r \sqrt{m^{2}-\frac{1}{4} \varepsilon^{2}}\right) / r$, with $\vec{\sigma}_{i}$ the $i$ component of the $\vec{\sigma}$ doublet. In order to avoid oscillatory solutions we demand that $\frac{1}{4} \varepsilon^{2}<m^{2}$. Another useful relation can be taken from the asymptotic forms of $\vec{\sigma}$ and $\boldsymbol{\theta}$ and from relations (28) and (31):

$$
\mathrm{E}=\frac{1}{2} \boldsymbol{\varepsilon} \cdot \mathbf{Q}+4 \pi \int r^{2} d r\left[U\left(\vec{\sigma}^{\dagger} \vec{\sigma}\right)+\vec{\sigma}^{\dagger^{\prime}} \vec{\sigma}^{\prime}\right]
$$

where $\mathbf{Q} \equiv\left(Q_{1}, Q_{2}, Q_{3}\right)$ as defined above. So, if this energy is less than the energy of the free particles the soliton is stable against decaying to free particles.

We will now try to find a solution corresponding to a quite large soliton in the thin-wall approximation. We will also take some general properties of the soliton. The more convenient method for solving the Euler-Lagrange equations is to regard the "matter" field $\sigma$ as a constant within a certain volume. In order to minimize the surface contribution to the total energy we regard the field configuration as spherical. The final scope of our work is to find an expression of the energy with respect to the charge, as we do in the global $S U(2)$ case. We will minimize the energy firstly with respect to the radius of the soliton and then with respect to the field value, as is the usual practice in the treatment of q-balls. We will use the following approximations: a)The thin-wall approximation for the matter field $\vec{\sigma}$. b)The assumption of small coupling constant, $g$. We will not use the approximate asymptotic relation for the energy given above as in 13 where the case of gauged $U(1)$ q-balls was treated, but the exact one, (equation 28). Let the matter have a spatially constant value $\sigma_{0}$ within a sphere of radius $R$. We will use eq. (31) so as to find $\theta_{i}(r)$. It is easy to find that:

$$
\theta_{i}(r)=\frac{\left(\varepsilon_{i}-\frac{g^{2} Q_{i}}{4 \pi R}\right) R \sinh (g \Sigma r)}{r \sinh (g \Sigma R)}, \quad r \leq R
$$


and:

$$
\theta_{i}(r)=\varepsilon_{i}-\frac{g^{2} Q_{i}}{4 \pi r}, \quad r \geq R
$$

where $\Sigma \equiv\left[(1 / 2) \vec{\sigma}^{\dagger} \vec{\sigma}\right]^{1 / 2}$. It is easy to see that the gauge field at large distances of the soliton origin has the form of a field generated from a spherically symmetric localized distribution carrying $S U(2)$ charge, without magnetic fields. Eq. 31 gives

$$
\varepsilon_{i}=\frac{g^{3} Q_{i} \Sigma}{4 \pi}\left(\frac{1}{g \Sigma R-\tanh (g \Sigma R)}\right) .
$$

Inserting $\varepsilon_{i}$ from the above equation in the relations 36 37 and substituting the result in the exact expression of the energy we find:

$$
\begin{array}{r}
E=\frac{g^{2} \mathbf{Q}^{2}}{8 \pi R}+\left\{3[-1+\exp (2 g R \Sigma)] g^{2} \mathbf{Q}^{2}-\right. \\
\left.32[-1+\exp (2 g R \Sigma)] \pi^{2} R^{4} U+32[1+\exp (2 g R \Sigma)] g \pi^{2} R^{5} \Sigma U\right\} \\
\{24 \pi R[1+g R \Sigma+\exp (2 g R \Sigma)(-1+g R \Sigma)]\}^{-1} .
\end{array}
$$

In the above expression the first term is the energy contribution of the gauge field outside the q-ball. The other terms give the energy of the interior of the soliton. We differentiate the above expression with respect to the radius of the configuration and we set the result equal to zero. In order to solve the transcendental equation that we take, we expand it in a power series of the coupling constant $g$. The equation that we have to solve is:

$$
-\frac{9 \mathbf{Q}^{2}}{8 \pi R^{4} \Sigma^{2}}+4 \pi R^{2} U-\frac{3 \mathbf{Q}^{2} g^{2}}{20 \pi R^{2}}=0
$$

The solution to the equation should also be expanded in a power series of $g$ :

$$
R=\frac{1}{2}\left(\frac{3}{\pi}\right)^{1 / 3} \frac{\left(2 \mathbf{Q}^{2} \Sigma^{4} U^{2}\right)^{1 / 6}}{\Sigma \sqrt{U}}+\frac{\mathbf{Q}^{2} \Sigma^{4} U g^{2}}{\Sigma \sqrt{U} 60 \pi\left(2 \mathbf{Q}^{2} \Sigma^{4} U^{2}\right)^{1 / 2}} .
$$

Inserting the above expression into the expression of energy and expanding again the energy with respect to $g$ we find

$$
\mathrm{E}=\left(\mathrm{Q}^{2} \frac{2 U}{\Sigma^{2}}\right)^{1 / 2}\left[1+\frac{g^{2}\left(\mathbf{Q}^{2}\right)^{1 / 3}\left(\frac{1}{4 \pi} \frac{3 \Sigma^{2}}{\sqrt{2 U}}\right)^{2 / 3}}{5}\right]
$$


The limit of the above expression when $g \rightarrow 0$ is what we expect from the global case. The final step is to minimize the energy with respect to the field $\Sigma$. Let $\Sigma_{0}$ be the value of the field minimizing the energy in the global case. Then the value that minimizes the energy in the local case is slightly different from the first one, as one can expect when the coupling constant is small and is given by:

$$
\Sigma=\Sigma_{0}\left(1-\frac{g^{2}}{5}\left(\frac{2 \mathbf{Q}^{2}}{3 \pi^{2}} \frac{\left(\varepsilon_{0}^{2}\right)^{2}}{\Sigma_{0}^{4}}\right)^{1 / 3}\left[\left[\frac{\partial^{2}}{\partial \Sigma^{2}}\left(\frac{U}{\Sigma^{2}}\right)\right]_{\Sigma=\Sigma_{0}}\right]^{-1}\right),
$$

where:

$$
\varepsilon_{0}^{2} \equiv \frac{2 U\left(\Sigma_{0}\right)}{\Sigma_{0}^{2}},
$$

the value of the "frequency" that minimizes the energy in the $g \rightarrow 0$ case. Let

$$
k \equiv\left[\left[\frac{\partial^{2}}{\partial \Sigma^{2}}\left(\frac{U}{\Sigma^{2}}\right)\right]_{\Sigma=\Sigma_{0}}\right] .
$$

If we substitute eq. 42 into 41 and keep only the $g^{2}$ terms of a Taylor expansion we take

$$
\mathrm{E}=\sqrt{\mathbf{Q}^{2} \frac{2 U}{\Sigma_{0}^{2}}}\left[1+\frac{3 \Sigma_{0}^{4 / 3} \kappa\left(\mathbf{Q}^{2}\right)^{1 / 3}+4\left[\frac{\left(\varepsilon_{0}^{2}\right)^{2} \mathbf{Q}^{2}}{\Sigma_{0}^{4}}\right]^{1 / 3}}{10 * 12^{1 / 3} \kappa \pi^{2 / 3} U^{1 / 3}} g^{2}\right] .
$$

In conclusion, we minimized the energy with respect to the radius of the field configuration (eq. 41) and the field $\vec{\sigma}$ (eq. 44). The energy seems to be larger than in the global case due to the terms depending on $g^{2}$. There is actually a slight decrease in the energy with the coupling constant. This result will be verified numerically. But the energy also depends on the charge. This total charge, $\sqrt{\mathbf{Q}^{2}}$, may be different in the local case from the global one. Intuitively, we expect that this charge (particle number) is smaller in the case of the local symmetry due to the electrostatic-type repulsion. This result will also be verified by numerical analysis.

\section{Numerical results}

The Euler-Lagrange equations of the matter and the gauge field are eqs. 26. 27. The energy and the soliton charge are given from the equations 28 and 31 


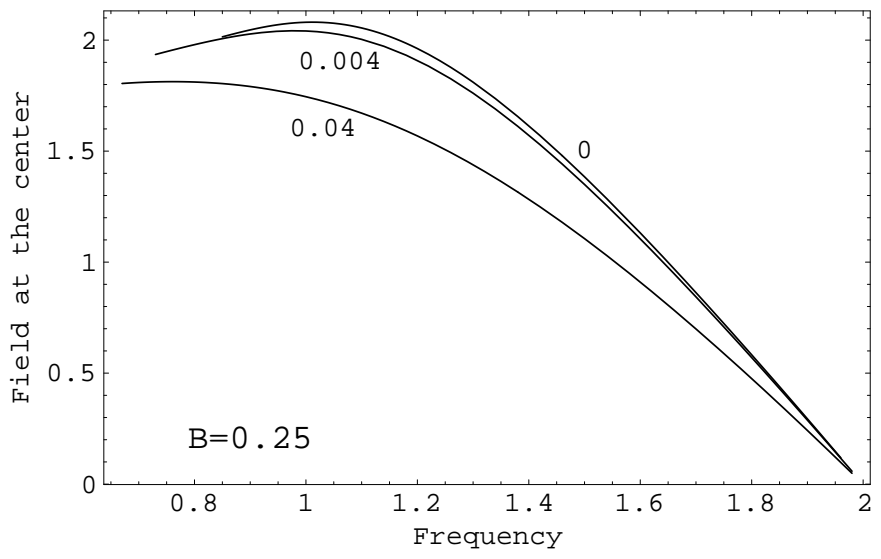

Figure 1: The central value of the field as a function of $\sqrt{\varepsilon^{2}}$ for three different values of the coupling constant with $B=0.25$.

respectively. In the global case we put $g=0$ and $\boldsymbol{\theta}=\boldsymbol{\varepsilon}$. The soliton charge is half the energy of the free particles with the same charge if the mass is unity as we have seen.

Figures 1, 2, 3 and 4 show the behavior of the soliton as a function of $\sqrt{\varepsilon^{2}}$. The square of the coupling constant takes the values $0,0.004$ and 0.04 . The first is the case of the global symmetry. We see that when $\sqrt{\varepsilon^{2}}$ approaches the critical (minimum) value, $\sqrt{\varepsilon_{0}^{2}}$, which holds for the thin-wall approximation, the "peak" of the soliton approaches the $2 / 3 B$ value according to eq. [17. and the energy and the charge of the soliton increase. These all agree with the idea that when the frequency approaches the critical, minimum, value, the contribution of the spatial field derivatives to the total soliton energy get smaller and we can talk for a large soliton with large energy contributions from the time dependence and the potential. When $\sqrt{\varepsilon^{2}} \rightarrow 2 m$ the size, energy and charge of the soliton decrease, tending to a flat field configuration covering the whole space with a very small value which corresponds to free particles. For the above reason the ratio soliton energy per free particles energy approaches unity. When the coupling constant differs from zero (i.e. when referring to the case of local symmetry) the soliton energy and charge are less than in the global case.

Figures 5, 8 give the central value, the energy of the soliton, the energy of free particles with the same charge and the ratio of the two energies. The potential now is less "deep", as the new value of the parameter $B$ is 0.75 . This has some interesting consequences. The minimum value of the frequency $(2 \sqrt{1-2 / 9 B})$ is now larger than in the case of $B=0.25$. So the ratio of the 


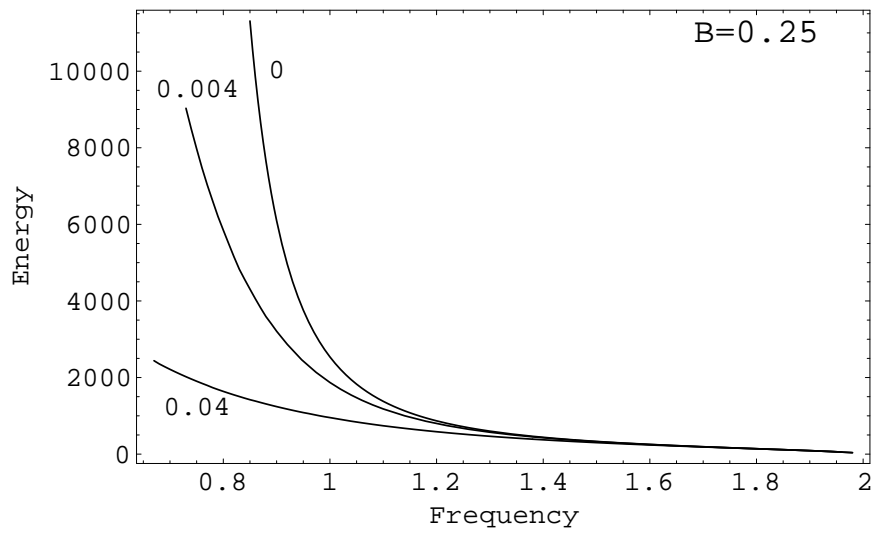

Figure 2: The energy of the soliton as a function of $\sqrt{\varepsilon^{2}}$ for three different values of the coupling constant with $B=0.25$.

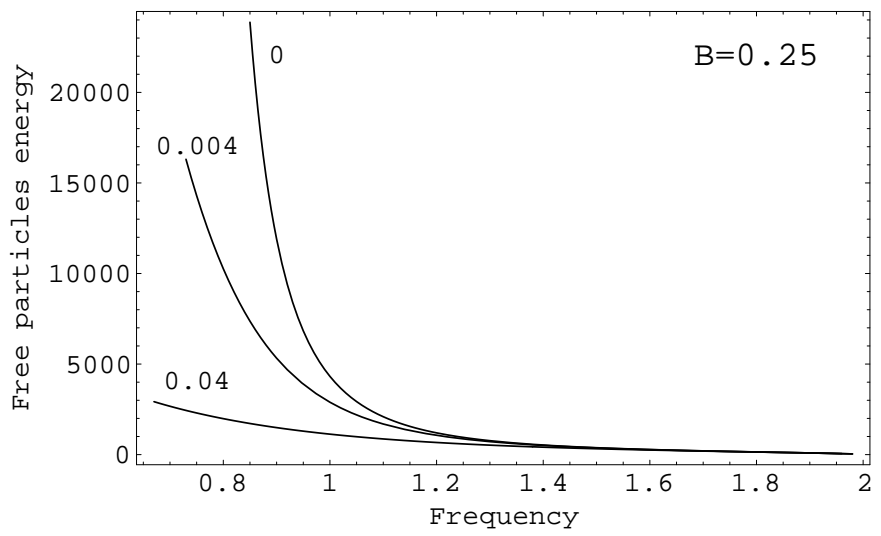

Figure 3: The energy of the free particles with charge equal to the soliton charge as a function of $\sqrt{\varepsilon^{2}}$ for three different values of the coupling constant with $B=0.25$. The charge of the soliton is half the value of the energy of the free particles. 


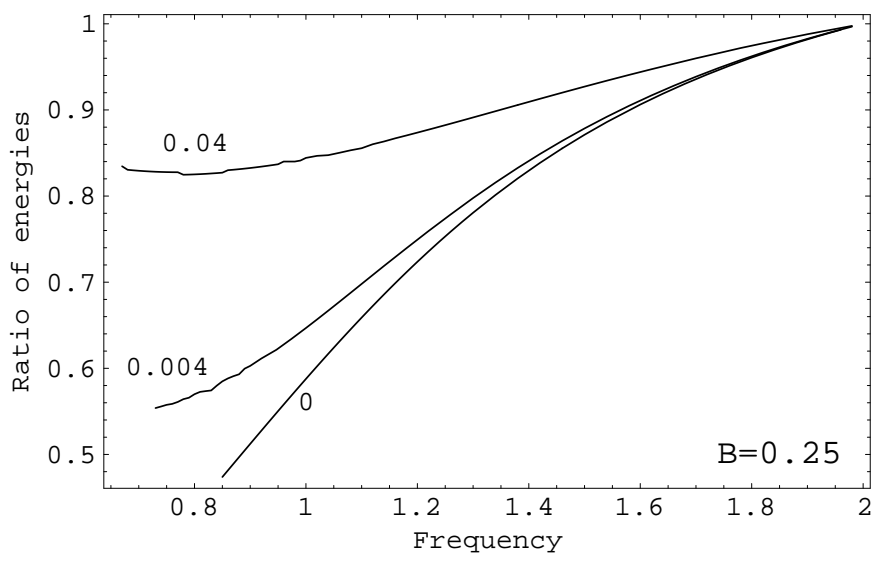

Figure 4: The soliton energy per the energy of free particles with equal charge as a function of $\sqrt{\varepsilon^{2}}$

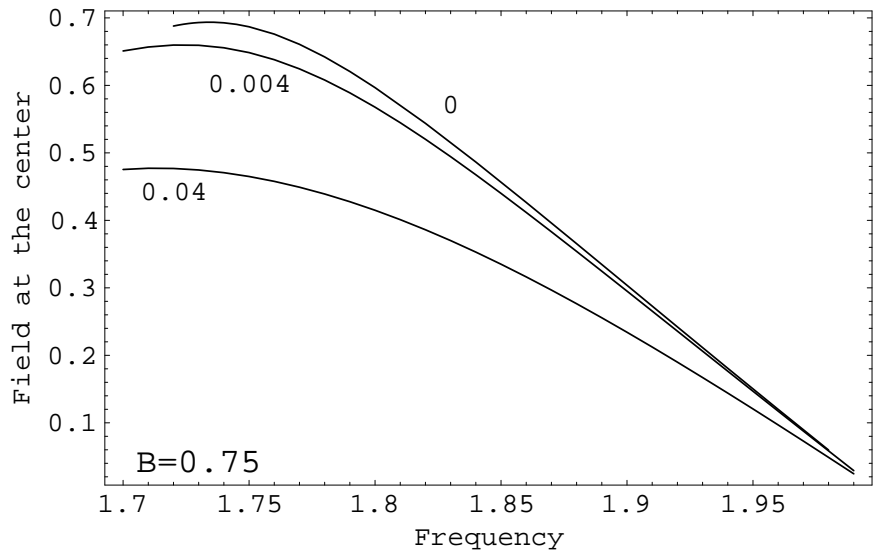

Figure 5: The central value of the field as a function of $\sqrt{\varepsilon^{2}}$ for three different values of the coupling constant with $B=0.75$. 


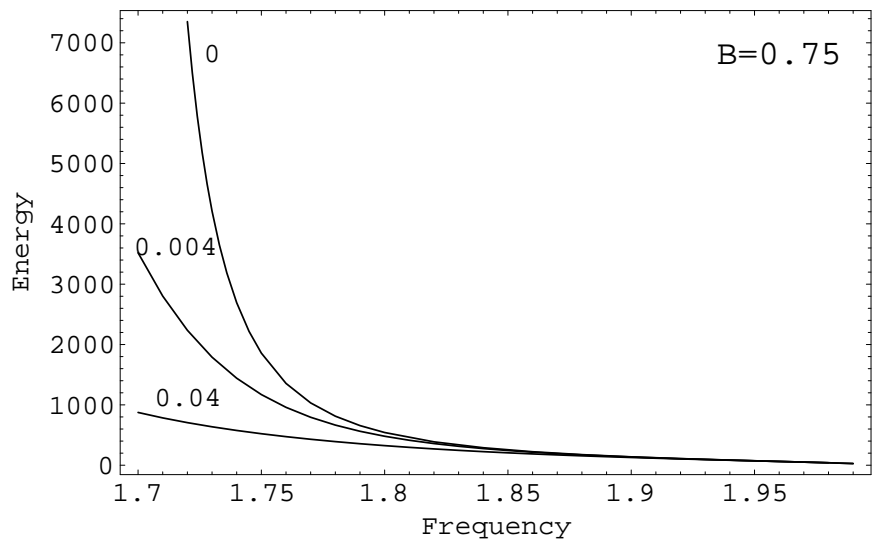

Figure 6: The energy of the soliton as a function of $\sqrt{\varepsilon^{2}}$ for three different values of the coupling constant with $B=0.75$.

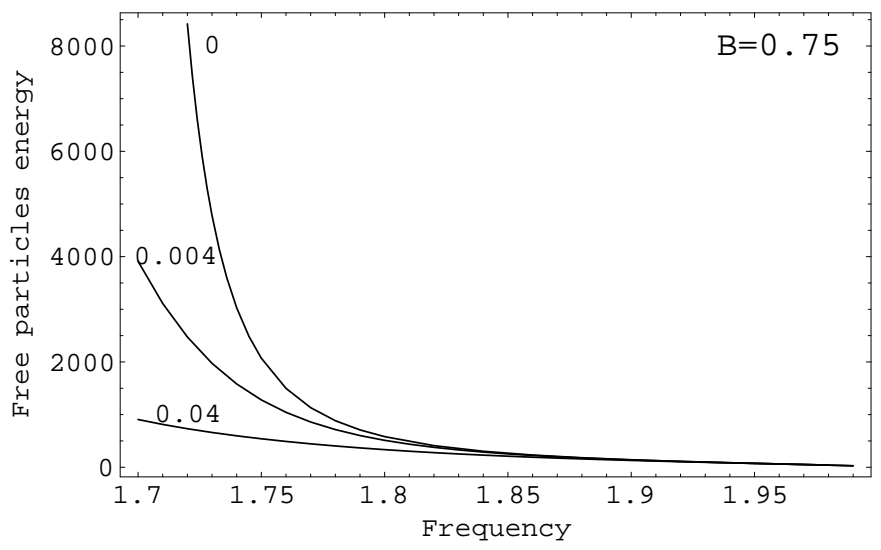

Figure 7: The energy of the free particles with charge equal to the soliton charge as a function of $\sqrt{\varepsilon^{2}}$ for three different values of the coupling constant with $B=0.75$. The charge of the soliton is half the value of the energy of the free particles. 


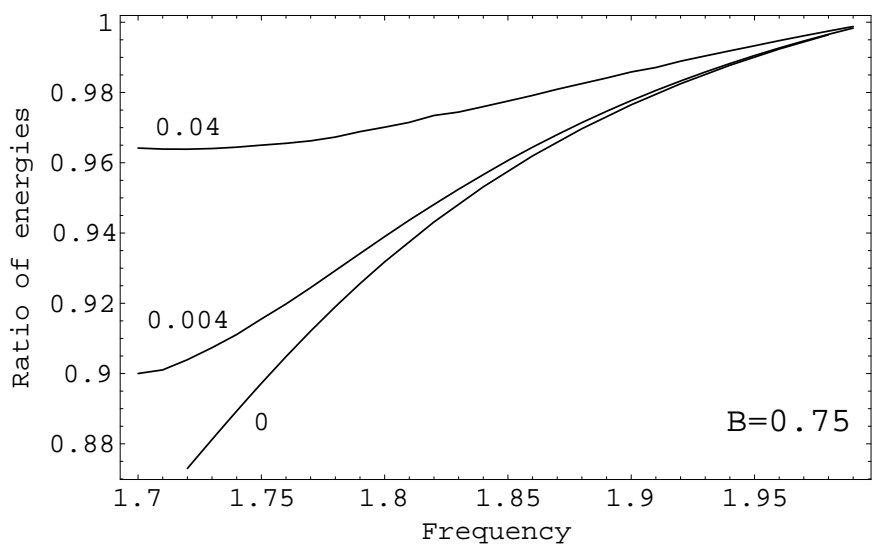

Figure 8: The soliton energy per the energy of free particles with equal charge as a function of $\sqrt{\varepsilon^{2}}$

soliton energy per the energy of the free particles is in general larger than in the case of $B=0.25$, because the minimum frequency is the lower limit of the above ratio.

In figures 9, 12 we study the behavior of the soliton when the coupling constant varies. When the coupling constant increases the soliton value decreases. This is an expected behavior due to the repulsion between the different parts of the soliton and the main reason for the decrease of the particle number (i.e. the soliton charge.) We will now explain the behavior of the soliton energy as a function of the coupling constant. Firstly the energy decreases with respect to the coupling constant due to the decrease of the value of the scalar field. Then, the energy seems to increase slightly due to the increase of the "electrostatic" energy contribution.

\section{Conclusions}

We found the energy and charge for a specific field configuration corresponding to the q-ball ansatz (eq. 6) in a Lagrangian of a spinor doublet of two scalar fields with a global $S U(2)$ symmetry. In the thin-wall approximation we found that, for potentials raising near the origin as $|\varphi|^{2}$, then slower than $|\varphi|^{2}$ and for large $|\varphi|$ faster than $|\varphi|^{2}$, q-ball-type solitons can be observed

at or near the minimum of the $\sqrt{U /|\varphi|^{2}}$ quantity. In this case the soliton energy takes a simple form and is smaller than the energy of the free par- 


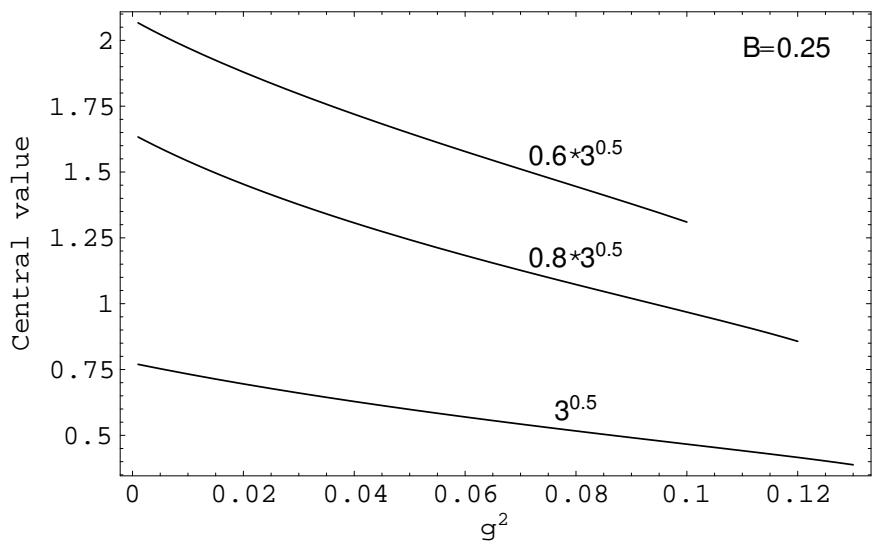

Figure 9: The central value of the field as a function of the coupling constant for three different values of the frequency with $B=0.25$.

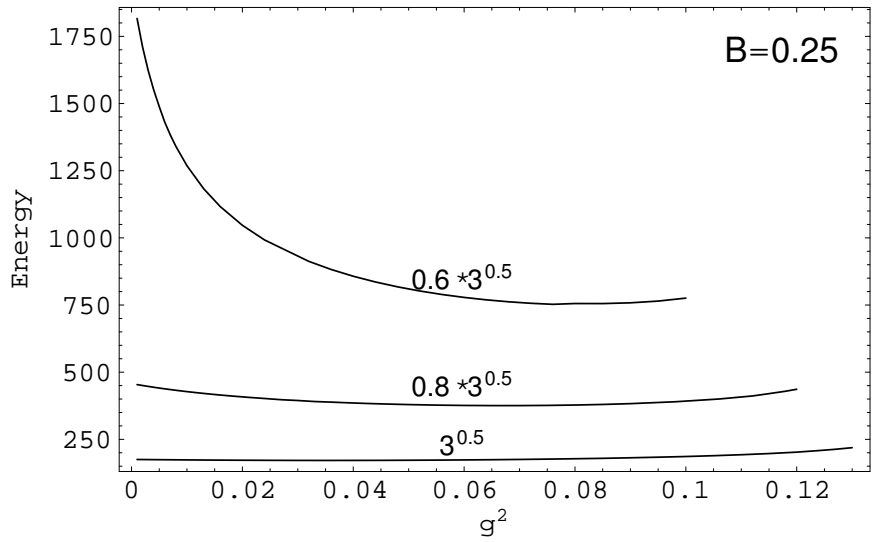

Figure 10: The energy of the soliton as a function of coupling constant for three different values of the frequency with $B=0.25$. 


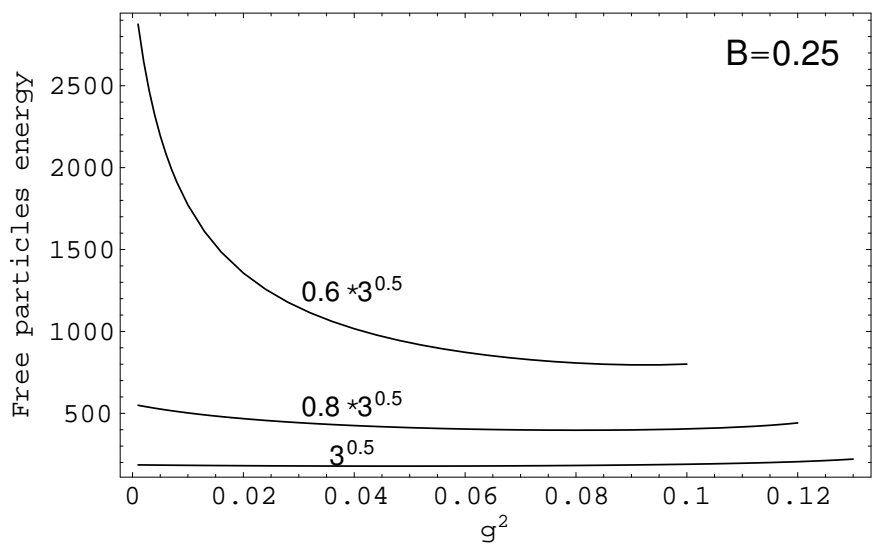

Figure 11: The energy of the free particles with charge equal to the soliton charge as a function of the coupling constant for three different values of the frequency with $B=0.25$. The charge of the soliton is half the value of the energy of the free particles.

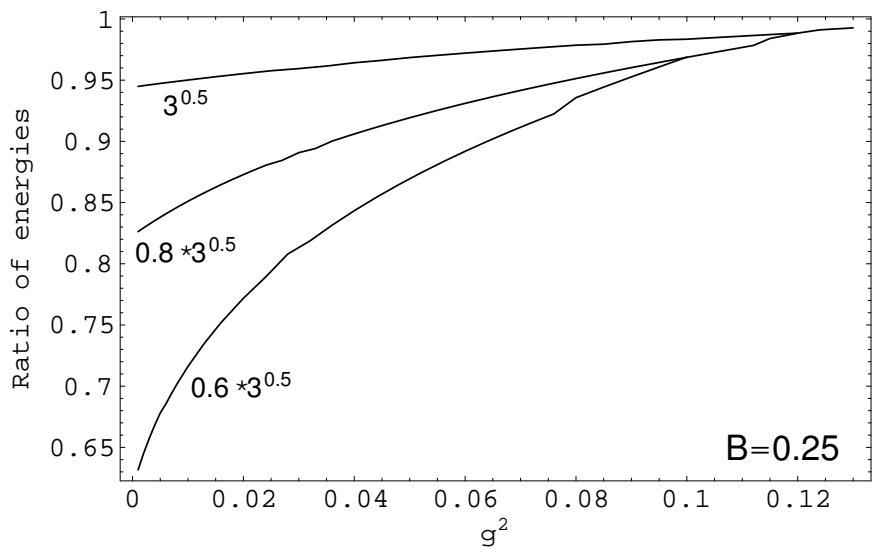

Figure 12: The soliton energy per the energy of free particles with equal charge as a function of the coupling constant with $B=0.25$. 
ticles with the same charge, provided that the above quantity is less than the mass of the free particles. In the thick-wall approximation we used the full Euler-Lagrange equation for the field and after solving it numerically we found that for a large region in the parameter space the soliton is prevented from fission into free particles thanks to the energy and charge conservation.

In the case of local $S U(2)$ symmetry we found that a localized field configuration is possible as a solution of the Euler-Lagrange equations. We found the energy of such a distribution as a function of charge. We minimized the energy with respect to the radius. Minimizing the energy with respect to the field value we found that this value is less than the corresponding to the global case due to the repulsion between the different parts of the charged soliton. For small values of the coupling constant and using numerical methods we found that a)there are localized and, thus, non-topological-soliton-type solutions to the equations of motion and b)their energy is less than the energy of the free particles. The comparison of the properties of the numerical solutions with different values of the parameters (coupling constant, parameters of the potential etc) helps us to fully understand the behavior of the soliton and the differences between the local and the global case.

\section{ACKNOWLEDGEMENTS}

I wish to thank A. Kehagias, G. Leontaris and N. D. Tracas for helpful discussions.

\section{References}

[1] G. Rosen, J. Math. Phys. 9, 996 (1968); 9, 999 (1968).

[2] S. Coleman, Nucl. Phys. B 262, 263 (1985).

[3] A. Cohen, S. Coleman, H. Georgi and A. Manohar, Nucl. Phys. B 272, 301 (1986).

[4] T.D. Lee and Y. Pang, Phys. Rept. 221, 251 (1992).

[5] A. Kusenko, Phys. Lett. B 406, 26 (1997).

[6] K. Enqvist and J. McDonald, Phys. Lett. B 425, 309 (1998).

[7] M. Axenides, E.G. Floratos, G.K. Leontaris and N.D. Tracas, Phys. Lett. B 447, 67 (1999). 
[8] G. Dvali, A. Kusenko and M. Shaposhnikov, Phys. Lett. B 417, 99 (1998).

[9] I. Affleck and M. Dine, Nucl. Phys. B 249, 361 (1985).

[10] M. Dine, L. Randall and S. Thomas, Nucl. Phys. B 458, 291 (1996).

[11] A.M. Safian, S. Coleman and M. Axenides, Nucl. Phys. B 297, 498 (1988).

[12] M. Axenides, E. Floratos and A. Kehagias, Phys. Lett. B 444, 190 (1998).

[13] K. Lee, J.A. Stein-Schabes, R. Watkins and L.M. Widrow, Phys. Rev. D 39, 1665 (1989).

[14] G.B. Cleaver, A.E. Faraggi, D.V. Nanopoulos and J.W. Walker, Nucl. Phys. B 620, 259 (2002).

[15] G.B. Cleaver, A.E. Faraggi, D.V. Nanopoulos, Int. J. Mod. Phys. A 16, 425 (2001).

[16] G.B. Cleaver, A.E. Faraggi, D.V. Nanopoulos and J.W. Walker, Mod. Phys. Lett. A 15, 1191 (2000). 